\title{
Cluster randomized trial in the general practice research database: 2 . Secondary prevention after first stroke (eCRT study): study protocol for a randomized controlled trial
}

\author{
Alex Dregan ${ }^{1 *}$, Tjeerd van Staa ${ }^{2}$, Lisa Mcdermott ${ }^{3}$, Gerard McCann², Mark Ashworth', Judith Charlton 1 , \\ Charles Wolfe', Anthony Rudd ${ }^{1}$, Lucy Yardley ${ }^{3}$ and Martin Gulliford ${ }^{1}$ for eCRT Research Team
}

\begin{abstract}
Background: The purpose of this research is to develop and evaluate methods for conducting pragmatic cluster randomized trials in a primary care electronic database. The proposal describes one application, in a less frequent chronic condition of public health importance, secondary prevention of stroke. A related protocol in antibiotic prescribing was reported previously.

Methods/Design: The study aims to implement a cluster randomized trial (CRT) using the electronic patient records of the General Practice Research Database (GPRD) as a sampling frame and data source. The specific objective of the trial is to evaluate the effectiveness of a computer-delivered intervention at enhancing the delivery of stroke secondary prevention in primary care. GPRD family practices will be allocated to the intervention or usual care. The intervention promotes the use of electronic prompts to support adherence with the recommendations of the UK Intercollegiate Stroke Working Party and NICE guidelines for the secondary prevention of stroke in primary care. Primary outcome measure will be the difference in systolic blood pressure between intervention and control trial arms at 12-month follow-up. Secondary outcomes will be differences in serum cholesterol, prescribing of antihypertensive drugs, statins, and antiplatelet therapy. The intervention will continue for 12 months. Information on the utilization of the decision-support tools will also be analyzed.
\end{abstract}

Discussion: The CRT will investigate the effectiveness of using a computer-delivered intervention to reduce the risk of stroke recurrence following a first stroke event. The study will provide methodological guidance on the implementation of CRTs in electronic databases in primary care.

Trial registration: Current Controlled Trials ISRCTN35701810

Keywords: Clinical trials, Cluster analysis, Electronic health records, Feasibility studies, Stroke, Secondary prevention

\section{Background}

Stroke represents a leading cause of avoidable morbidity and mortality in the UK with 130,000 stroke events per year [1]. Patients who have had a first stroke have a considerably elevated risk of a second stroke as well as increased risk of myocardial infarction, lower limb ischemia, and other

\footnotetext{
* Correspondence: alexandru.dregan@kcl.ac.uk

'King's College London, Department of Primary Care and Public Health Sciences, Division of Health and Social Care Research, Capital House, 42 Weston St, London SE1 3QD, UK

Full list of author information is available at the end of the article
}

vascular events $[2,3]$. The outcomes for those who have a recurrent stroke are generally worse than after a first stroke, with patients likely to experience increased disability and higher healthcare costs over the longer term [4]. An important objective of preventive medical care is to reduce the risk of recurrence of stroke and progression of vascular disease.

Secondary prevention after a first stroke should include lifestyle interventions to reduce overweight, increase exercise, reduce alcohol intake and smoking, as well as attention to control of risk factors for cardiovascular disease

\section{Ciomed Central}

(c) 2012 Dregan et al.; licensee BioMed Central Ltd. This is an Open Access article distributed under the terms of the Creative Commons Attribution License (http://creativecommons.org/licenses/by/2.0), which permits unrestricted use, distribution, and reproduction in any medium, provided the original work is properly cited. 
including blood pressure (BP), serum cholesterol, and antithrombotic treatment where appropriate $[1,5,6]$. National audits have revealed 'major deficiencies' in stroke secondary prevention with $40 \%$ or fewer subjects achieving a target systolic blood pressure of $<140 \mathrm{~mm} \mathrm{Hg}$ at 6 months post stroke [7]. Stroke secondary prevention has been specifically incentivized within the Quality and Outcomes Framework (QOF) of the general practice contract [8] with targets for treatment and control of blood pressure $(<150 / 90 \mathrm{~mm} \mathrm{Hg})$ and cholesterol $(\leq 5 \mathrm{mmol} / \mathrm{L})$, antiplatelet or anticoagulant therapy for eligible patients, and flu immunization [9]. However, these targets are flexible with some $8 \%$ of patients being excluded as 'exceptions' and intermediate outcomes only requiring measurement within the last 15 months to less stringent targets. While the QOF strategy represents a 'control' intervention, a more rigorous, evidence-based intervention strategy has been recommended in the National Clinical Guidelines for Stroke from the ICSWP [1], now in their third edition [10]. These more stringent recommendations include: BP should be controlled to $<130 / 80 \mathrm{~mm} \mathrm{Hg}$ in most patients; all subjects with total cholesterol $>4 \mathrm{mmol} / \mathrm{L}$ should be treated with a statin unless there is evidence of hemorrhagic stroke; and antiplatelet therapy should be prescribed where appropriate.

The responsibility for delivering effective secondary prevention, and managing long-term problems associated with stroke, lies initially with the primary care team. Considerable efforts have therefore been made to enhance the quality of preventive medical care in general practice and to increase the achievement of clinical targets for secondary prevention of stroke. The preferred design to evaluate interventions aimed at changing practitioner behavior and improve practice during contact with patients is the cluster randomized trial (CRT) [11-13]. A CRT design is appropriate when the unit of intervention is a healthcare practitioner or a healthcare organization such as a general practice. Well designed CRTs may capitalize on the effects of within-group learning, such as may occur when clinical decision support systems are deployed to primary care teams, while minimizing cross-group contamination [14]. Van Staa et al. [15] suggested that the utilization of large databases of electronic health records may facilitate the implementation of pragmatic trials at low cost and with reduced data collection burden for the clinician, health service, or the patient. Use of electronic patient records facilitates the identification of all patients with a specific condition registered at a particular practice [14], potentially increasing the external validity of the study findings [15]. Therefore the proposed study will use a pragmatic CRT to compare routine practice under QOF with a more rigorous secondary prevention strategy as recommended by the ICSWP.
This proposal will build on our experience of implementing a CRT to enhance secondary prevention after stroke utilizing the South London Stroke Register [16,17]. In a recent cluster trial using stroke register data to inform stroke care, Wolfe et al. [18] found no improvement in risk factor management at 12-month follow-up. The trial was implemented in an inner-city locality with a modest sample size and collection of outcome data collection through interaction with participants could have primed the controls about the aims of the trial potentially leading to positive lifestyle changes or social desirability bias.

The present trial aims to compare routine practice under QOF with a more rigorous secondary prevention strategy as recommended by the ICSWP. The planned trial will extend to practices throughout the UK. Individual participants will not be aware of the aims of the intervention reducing the risk of contamination.

\section{Methods/Design \\ Objective}

The primary objective of the proposed study is to evaluate whether a more intensive secondary prevention strategy, delivered at the level of general practice and based on evidence-based recommendations to control risk factors, gives improved intermediate clinical outcomes and processes of care following first stroke.

\section{Study design}

The effectiveness of a computer-delivered intervention to promote secondary prevention of stroke will be tested in a pragmatic cluster randomized trial with two trial arms: usual care vs. computer-delivered recommendations for secondary prevention of stroke. The general practice is chosen as the unit of allocation because the intervention is implemented at the level of the general practice. The duration of intervention is 12 months and the first practices were allocated in April 2012.

\section{Study setting and population}

The study will take place in family practices in England, Scotland, and Wales that contribute data to General Practice Research Database (GPRD) which is part of the Clinical Practice Research Datalink (CPRD). The age and sex distribution of the GPRD registered population is similar to the distribution of the UK population [19]. The study sample is represented by GPRD practices that provide written consent to participate in the trial, in areas that provide research governance approval of the study. Study practices are recruited through a letter of invitation sent from GPRD/MHRA. The intervention is delivered through software known as DXS, and only those GPRD practices that use DXS Point-of-Care will be eligible to participate in the trial. 


\section{Allocation}

GPRD practices will be allocated to the two trial arms in equal proportions. The allocation is by minimization controlling for region in England (North (North-East and North-West), Midlands (East and West Midlands), South-East (South-East and East of England), SouthWest, and London) and country in the UK (Scotland, Wales, England) and list size (number of registered patients). This list size was dichotomized for the minimization using 7,500 as the cut-point. The allocation is performed at King's College London using anonymized practice identifiers supplied by the recruitment team at GPRD/MHRA.

\section{Intervention}

The intervention comprises a series of electronic prompts that promote adherence with evidence-based recommendations for secondary prevention of stroke and vascular disease following the National Guidelines $[5,6]$. The development and implementation of the intervention was informed by qualitative research for this project $[16,17]$ which was adapted to recent guidelines. A key element of the intervention will be a series of electronic prompts that operationalize the ICSWP recommendations. Control practices will continue with usual care. The intervention will be implemented for 12 months at each practice. A detailed description of the development and design of the electronic prompts is provided in McDermott et al. [20]

\section{Intervention implementation}

Electronic prompts will be installed remotely at intervention general practices. Electronic prompts will be activated during eligible consultations. Consultations will be eligible if the patient is aged $\geq 18$ years and is included on the practice stroke register. During consultations with patients who have previously had a stroke, primary care professionals will see the prompts which remind them of evidence-based guidelines for secondary prevention of stroke. The main focus is on targets for control of blood pressure and cholesterol levels, appropriate prescribing of aspirin and antiplatelet drugs, and enhanced recording of stroke type (ischemic or hemorrhagic). The prompts will also provide GPs with supporting information, links to evidence that supports the recommendations, and options to print patient information. The decision on whether to follow the treatment suggestions included in the prompts will be at the discretion of the GP. The GP will also be able to terminate display of the prompt at any time. At the start of the study, a letter containing instructions on the use of the prompts will be sent to general practices in the intervention trial arm for circulation to participating general practitioners. All control practices will be sent a letter reminding them to record any relevant consultation for eligible stroke patients and to notify any adverse events. Three months into the study, all intervention practices will receive an email reminder containing instructions about the prompts including aims and implementation.

\section{Outcome evaluation}

The evaluation of outcomes will be through analysis of routinely-collected GPRD data during a defined study period, while historical information will be used to assess the baseline characteristics of the study patients. Information routinely collected into GPRD for all registered patients includes medical history, use of medicines, hospitalizations and other resource use, smoking history, laboratory tests, letters from specialists or hospitals. GPRD also now links patients in GPRD to the English Hospital Episode Statistics, with detailed information on date, duration, and reason for hospitalization. Availability of data for all registered patients has potential to minimize biases from subject selection/recruitment.

Electronic records will be included in analyses for all patients on the practice's stroke register. Participants that were diagnosed with transient ischemic attacks only will be excluded. The primary outcome measure is the mean systolic BP after 12 months. Modeling will be used to utilize BP values measured over time during the intervention period. [21] Secondary outcomes will be mean diastolic blood pressure; mean cholesterol concentration; proportion of patients whose eligibility for anticoagulants/antiplatelet drugs is defined; proportion of eligible patients that receive anticoagulant/antiplatelet drugs; prescription adherence with prescribed medicines; occurrence and hospitalization with vascular events including TIA/stroke, myocardial infarction, new-onset angina, and mortality. In light of recent changes in guidelines for anti-platelet treatment, an additional outcome will be the proportion of patients that received clopidogrel as the first drug of choice. Recurrent stroke events will be validated through anonymized records obtained from practices. Linked HES data may be used to evaluate hospital utilization. The method of Generalized Estimating Equations will be implemented to allow for clustering by practice, providing an analytical framework for both binary and continuous outcomes [22]. Last value carried forward and multiple imputation will be used to deal with missing values.

\section{Process evaluation}

The study will also evaluate the obstacles, barriers, and facilitators to implementation of intervention research within the GPRD. We will conduct an intervention evaluation study towards the end of the trial including both staff at GPRD and study practices via an electronic questionnaire to ensure the anonymity of practices is 
maintained. General practices in the 'control' arm of the study will only be asked to complete two questions on 'views and opinions'. In addition, practices who agreed to a telephone interview at the time of consent for the trial will be contacted for a telephone interview at the end of the study to discuss GPs' opinions and feedback of experiences of taking part in the trial.

\section{Sample size calculation}

The sample size calculation is based on a comparison between the trial intervention and usual care, represented by the targets included in the quality and outcomes framework (QOF) for stroke. Thus the QOF recommends a blood pressure target for stroke patients of $150 / 90 \mathrm{~mm}$ $\mathrm{Hg}$ while the trial prompts, following the ICSWP guidelines, recommend a target of 130/80 $\mathrm{mm} \mathrm{Hg}$. The agestandardized incidence of first stroke is between 1.74 and 2.16 per 1,000 in England [2,23]. In 375 GPRD practices in 2006, there were approximately 14.5 first strokes per practice per year (ISAC protocol 07-027). We propose to include for analysis all subjects whose first stroke was in the 2 years preceding the intervention start date, giving about 29 subjects per practice eligible for analysis. If the standard deviation of systolic BP in these subjects is 19 $\mathrm{mm} \mathrm{Hg}[24,25]$ with an ICC by general practice of 0.032 [26], with a two-sided alpha $=0.05$, power $=0.8$, then to detect a $2.75 \mathrm{~mm} \mathrm{Hg}$ difference in systolic blood pressure, about 99 practices will be required with 50 receiving the active intervention. This represents an effect size of 0.14 . In reality, the number of patients per practice will be variable and the coefficient of variation of practice size may be approximately 0.65 [27]. This means that the effective number of practices is approximately 83 and the detectable difference in systolic BP will be approximately $3 \mathrm{~mm}$ Hg. Analysis of GPRD data for stroke shows a high proportion of stroke patients had blood pressure recordings in 2006 with a mean of three blood pressure records per patient.

\section{Ethics}

The study protocol and associated documents have received approval/favorable opinion from the National Research Ethics Service Committee London - South West (REC number: 08/H0801/113) and from the R\&D from participating Primary Care Trusts (PCTs) or Health Boards (HB). Protocol amendments have received REC approval and approval/favorable opinion from participating PCTs and HBs. A senior partner at each participating general practice will be asked to give written informed consent to take part in the study [27].

\section{Discussion}

This study, together with a previously reported trial in antibiotic prescribing [28], will provide evidence concerning the feasibility of implementing CRTs using electronic databases and its effectiveness in preventing stroke recurrence and associated vascular events. The methodology developed through this project will have wide potential for application in future research with primary care electronic databases. A key output from the research will therefore be a set of methodological guidelines that will identify and analyze the component tasks of implementing a cluster trial through electronic patient records.

Cluster trials are susceptible to bias and the implementation of a cluster trial within an electronic database offers the opportunity to evaluate such biases. For example, in practices allocated to the intervention the intervention may be associated with increased recruitment rate [29]. Also, the behavior of professionals at control practices may be modified through their participation in the study even though they are not exposed to the intervention [28].

These potential biases may be evaluated by comparing number of stroke patients at intervention practices prior to and after the intervention and also comparing changes in secondary prevention activity between nonparticipating and participating practices.

\section{Trial status}

Practice recruitment stage.

\section{Abbreviations}

CRT: Cluster randomized trials; GPRD: General Practice Research Database; HB: Health boards; HES: Hospital episode statistics; ICSWP: Intercollegiate Stroke Working Party; ITT: Intention to treat; KCL: King's College London; MHRA: Medicines and Healthcare products Regulatory Agency; NHS: National Health Service; QOF: Quality and Outcomes Framework; PCT: Primary care trusts; REC: Research and Ethics Committee.

\section{Competing interests}

The authors declare that they have no competing interests.

\section{Authors' contributions}

MG conceived of the study; AD drafted the protocol; $A D$ and MG contributed to the implementation of the study, the allocation process and the analysis plan; TVS, CW, AR, MA, LY, and JC and contributed to the design and implementation of the study and contributed to the protocol; GM was responsible for developing recruitment procedures; LM and LY were responsible for developing the trial interventions. All authors read and approved the final manuscript.

\section{Acknowledgements}

This research was supported by the Wellcome Trust and Research Councils' Joint Initiative in Electronic Patient Records and Databases in Research. AR is supported by the Guy's and St Thomas' NHS Trust / King's Health Partners research programmed activities scheme.

The authors thank the staff of DXS (UK) Ltd for facilitating the implementation of the intervention through DXS Point-of-Care. The eCRT Research Team also includes Paul Little, University of Southampton, Michael Moore, University of Southampton. Data Monitoring Committee: Sarah Meredith (Chair), Sally Kerry, Elizabeth Murray, Kate Huckett.

Trial Steering Committee: Jonathan Mant, Andrew Haywood, Nanik Pursani, John Robson. 


\section{Author details}

'King's College London, Department of Primary Care and Public Health Sciences, Division of Health and Social Care Research, Capital House, 42 Weston St, London SE1 3QD, UK. ${ }^{2}$ Clinical Practice Research Datalink, Medicines and Healthcare products Regulatory Agency, London, UK. ${ }^{3}$ School of Psychology, University of Southampton, Southampton, UK.

Received: 10 May 2012 Accepted: 26 September 2012

Published: 3 October 2012

\section{References}

1. Party ISW: National Clinical Guidelines for Stroke. 2nd edition. London: Royal College of Physicians; 2004.

2. Mant J, Wade D, Winner S: Health care needs assessment: stroke. In Health care needs assessment: the epidemiologically based needs assessment reviews. 2nd edition. Edited by Stevens A, Raftery J, Simpson S. Oxford: Radcliffe Medical; 2004

3. Warlow CP, Van Gijn J, Dennis MS, Wardlaw JM, Bamford JM, Hankey GJ, Sandercock PAG, Rinkel G, Langhorne P, Sudlow C, Rothwell P: Stroke: practical management. 3rd edition. Oxford: Blackwell Publishing; 2011.

4. Mohan KM, Crichton SL, Grieve AP, Rudd AG, Wolfe CD, Heuschmann PU: Frequency and predictors for the risk of stroke recurrence up to 10 years after stroke: the South London Stroke Register. J Neurol Neurosurg Psychiatry 2009, 80:1012-1018.

5. National Institute for Health and Clinical Excellence: Stroke: diagnosis and initial management of acute stroke and transient ischemic attack (TIA) London: NICE; 2008.

6. National Institute for Health and Clinical Excellence: Clopidogrel and modified-release dypiridamole for the prevention of occlusive vascular events (review of NICE technology appraisal guidance 90). Technology appraisal guidance 2010. London: NICE; 2010.

7. Rudd AG, Lowe D, Hoffman A, Irwin P, Pearson M: Secondary prevention for stroke in the United Kingdom: results from the National Sentinel Audit of Stroke. Age Ageing 2004, 33:280-286.

8. Doran T, Fullwood C, Gravelle H, Reeves D, Kontopantelis E, Hiroeh U, Roland M: Pay-for-performance programs in family practices in the United Kingdom. New Engl J Med 2006, 355:375-384.

9. Employers NHS: Quality and outcomes framework guidance 20011/12. London: NHS Confederation; 2012.

10. Intercollegiate Stroke Working Party: National Clinical Guidelines for Stroke. Draft for consultation. 3rd edition. London: Royal College of Physicians; 2008.

11. Glynn RJ, Brookhart MA, Stedman M, Avorn J, Solomon D: Design of cluster-randomized trials of quality improvement interventions aimed at medical care providers. Med Care 2007, 45:\$38-\$43.

12. Donner A, Klar N: Design and analysis of cluster randomisation trials in health research. London: Arnold; 2000.

13. Eldridge S, Karry S: A practical guide to cluster randomised trials in health service research. UK: Wiley \& Sons; 2012.

14. Love TE, Cebull RD, Einstadter D, Jain AK, Miller H, Harris CM, Greco PJ, Husak SS, Dawson NV, DIG-IT Investigators: Electronic medical recordassisted design of a cluster randomised trial to improve diabetes care and outcomes. J Gen Intern Med 2008, 23:383-391.

15. Van Staa T, Goldacre B, Gulliford M, Cassell J, Pirmohamed M, Taweel A, Delaney B, Smeeth L: Pragmatic randomised clinical trials using routine electronic health records: putting them to the test. BMJ 2012, 344:e55.

16. Redfern J, McKevitt C, Wolfe CD: Development of complex interventions in stroke care: a systematic review. Stroke 2006, 37:2410-2419.

17. Redfern J, Wolfe CD, Rudd AD, McKevitt C: Stop stroke: development of an innovative intervention to improve risk factor management after stroke. Patient Educ Couns 2008, 72:201-209.

18. Wolfe CDA, Redfern J, Rudd AG, Grieve AP, Heuschmann PU, McKevitt C: Cluster randomised controlled trial of a patient and general practitioner intervention to improve the management of multiple risk factors after stroke. Stroke 2011, 41:2470-2476.

19. Lawson DH, Sherman V, Hollowell J: The general practice research database. Scientific and Ethical Advisory Group. QJM 1998, 91:445-452.

20. McDermott L, Yardley L, Little P, Ashworth M, Gulliford M, eCRT Research Team: Developing a computer delivered, theory based intervention for guideline implementation in general practice. BMC Fam Pract 2010, 11:90.
21. Dregan A, Toschke MA, Wolfe CD, Rudd A, Ashworth M, Gulliford MC, eCRT Research team: Utility of electronic patient records in primary care for stroke secondary prevention trials. BMC Publ Health 2011, 11:86.

22. Ukoumunne OC, Carlin JB, Gulliford MC: A simulation study of odds ratio estimation for binary outcomes from cluster randomized trials. Stat Med 2007, 26:3415-3428.

23. Stewart JA, Dundas R, Howard RS, Rudd AG, Wolfe CDA: Ethnic differences in incidence of stroke: prospective study with stroke register. BMJ 1999, 318:967-971.

24. Toschke AM, Gulliford MC, Wolfe CD, Rudd AG, Heuschmann PU: Antihypertensive treatment after first stroke in primary care: results from the General Practitioner Research Database. J Hypertens 2011, 29:154-160.

25. PROGRESS Collaborative Group: Randomised trial of a perindopril-based blood-pressure-lowering regimen among 6105 individuals with previous stroke or transient ischaemic attack. Lancet 2001, 358:1033-1041.

26. Adams G, Gulliford MC, Ukoumunne OC, Eldridge S, Chinn S, Campbell MJ: Patterns of intra-cluster correlation from primary care research to inform study design and analysis. J Clin Epidemiol 2004, 57:785-794.

27. Eldridge SM, Ashby D, Kerry S: Sample size for cluster randomized trials: effect of coefficient of variation of cluster size and analysis method. Int J Epidemiol 2006, 35:1292-1300.

28. Gulliford MC, van Staa T, McDermott L, Dregan A, McCann G, Ashworth M, Charlton J, Grieve AP, Little P, Moore MV, Yardley L, electronic Cluster Randomised Trial Research Team eCRT Research Team: Cluster randomised trial in the General Practice Research Database: 1. Electronic decision support to reduce antibiotic prescribing in primary care (eCRT study). Trials 2011, 12:115.

29. Hahn S, Puffer S, Torgerson DJ, Watson J: Methodological bias in cluster randomised trials. BMC Med Res Methodol 2005, 5:10.

doi:10.1186/1745-6215-13-181

Cite this article as: Dregan et al:: Cluster randomized trial in the general practice research database: 2 . Secondary prevention after first stroke (eCRT study): study protocol for a randomized controlled trial. Trials 2012 13:181.

\section{Submit your next manuscript to BioMed Central and take full advantage of:}

- Convenient online submission

- Thorough peer review

- No space constraints or color figure charges

- Immediate publication on acceptance

- Inclusion in PubMed, CAS, Scopus and Google Scholar

- Research which is freely available for redistribution

Submit your manuscript at www.biomedcentral.com/submit
C Biomed Central 